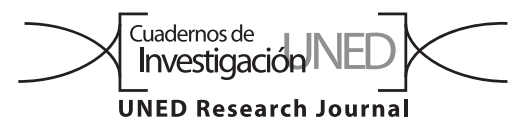

\title{
Los primeros 10 años de Cuadernos de Investigación UNED: bibliometría de temas, autores e instituciones
}

\author{
Julián Monge-Nájera \& Carolina Seas \\ Universidad Estatal a Distancia, Vicerrectoría de Investigación, 2050 San José, Costa Rica; julianmonge@gmail.com, cseas@uned.ac.cr
}

Recibido 02-V-2019 • Corregido 24-V-2019 • Aceptado 30-V-2019

\begin{abstract}
The first 10 years of UNED Research Journal: bibliometrics of subjects, authors and institutions". Introduction: Costa Rica is the Central American country with the most bibliometric studies, however, very few deal with the historical trends of particular academic journals. Objective: We analyze the statistical trends of the first decade of the journal Cuadernos de Investigación UNED / UNED Research Journal, considering four topics: who publishes, what they publish, how trends have varied over time and what we can expect in the future. Results: The number of tables per article was high at the beginning, then lower, usually between 2 and 3 . The number of figures per article ranges from 2 to 5 . The articles are becoming shorter over time. The number of authors per article ranges from 2 to 3 , one third to one half of them, women. The six authors with the highest total number of articles are from the Universidad Estatal a Distancia (UNED) and Universidad de Costa Rica (UCR); half of them are women. Articles on Costa Rica predominate, followed distantly by the rest of Latin America, Africa and Asia. Articles from Costa Rica have quadrupled over time. The institutions that predominate as sources of articles are the UNED, UCR and UNA. UNED has been stable in its contribution, UCR seems to increase its output every 5 years, and Universidad Nacional Autónoma de México (UNAM) has decreased over time. Those who publish about Costa Rica are Costa Rican authors. The increase of articles in Spanish is more marked and constant than articles in English. Conclusion: The historical patterns match other Costa Rican scientific journals, like Biología Tropical that, even though published in a tiny country, are inserted in the much wider world of international science.
\end{abstract}

Key words: open access, scientific journals, historical trends, scientific publication, Latin America.

Aunque Costa Rica es el país centroamericano más estudiado desde el punto de vista bibliométrico, existen relativamente pocos estudios sobre las tendencias históricas de revistas académicas particulares. El estudio cuantitativo de la ciencia costarricense inició en 1988, con un artículo de Monge-Nájera y Díaz (1988) sobre la Revista de Biología Tropical, el cual es además el único hasta ahora que ha usado pruebas estadísticas de
RESUMEN: Introducción: Costa Rica es el país centroamericano sobre el que se han hecho más estudios bibliométricos, sin embargo, muy pocos son sobre las tendencias históricas de revistas académicas particulares. Objetivo: Analizamos las tendencias estadísticas de la primera década de la revista Cuadernos de Investigación UNED / UNED Research Journal, considerando cuatro temas: quiénes publican, qué publican, cómo han variado las tendencias a lo largo del tiempo y qué podemos esperar en el futuro. Resultados: La cantidad de tablas solo fue alta al inicio, oscilando luego entre 2 y 3 tablas por artículo. La cantidad de figuras por artículo oscila entre 2 y 5 . Los artículos han tendido a acortarse. La cantidad de autores por artículo oscila entre 2 y 3 , siendo mujeres entre un tercio y la mitad. Las seis personas con mayor cantidad total de artículos publicados son de la Universidad Estatal a Distancia (UNED), seguida de la Universidad de Costa Rica (UCR); la mitad de personas de alta productividad son mujeres. Predominan los artículos sobre Costa Rica, seguidos de lejos por el resto de América Latina, África y Asia. Los artículos provenientes de Costa Rica se ha cuadriplicado en esta primera década. En cuanto al país de los autores, Costa Rica domina fuertemente. Las instituciones que predominan como fuentes de artículos son UNED, UCR y Universidad Nacional (UNA). La UNED ha sido estable, la UCR parece aumentar cada 5 años, y la Universidad Nacional de México ha disminuido su aporte anual. Quienes publican sobre Costa Rica son autores costarricenses. El aumento de los artículos en español es más marcado y constante que el de los artículos en inglés. Conclusión: Los patrones históricos coinciden con el de otras revistas científicas costarricenses como Biología Tropical que, aunque publicadas en un país pequeño, se insertan en el mundo mucho más amplio de la ciencia internacional.

Palabras clave: acceso abierto, revistas científicas, tendencias históricas, publicación científica, latinoamérica. hipótesis para medir la significancia de los patrones. Ese estudio halló que temáticamente dominaban la taxonomía animal, la biomedicina y la ecología; y que las instituciones extranjeras, y el inglés, representaban la mitad de los artículos. Dos años después, el estudio de Barrientos y Monge-Nájera (1990) analizó la revista agrícola Turrialba, hallando que dominaban los artículos sobre fisiología y patología vegetales, suelos, y técnicas agronómicas, con 
marcado tinte local, pues cada región se especializaba en ciertos temas.

Fuera del área de biología, sobresale el estudio de Carvajal y Matamoros (2012) sobre la Revista Costarricense de Psicología, el cual concluye que, a pesar de ser la psicología costarricense una especialidad numéricamente dominada por las mujeres, ellas son minoría entre quienes publican, y que en lugar de investigación científica, la revista, en sus primeros 30 años, publicó fundamentalmente resúmenes de libros con un enfoque "hermenéutico", el cual es considerado pseudocientífico (ver Bunge, 2007).

Ese mismo año apareció un estudio sobre los primeros 60 años de la Revista de Biología Tropical, el cual concluyó que, pese al pequeño tamaño de Costa Rica, su producción se compara favorablemente con la de países mucho más grandes como Brasil, México y Argentina; que el tema dominante es la zoología; y que cerca de la mitad de los estudios provenían de colaboración entre instituciones y se redactaba en lengua inglesa (NielsenMuñoz, Azofeifa-Mora, \& Monge-Nájera, 2012). Otro estudio, cuatro años después, obtuvo básicamente los mismos resultados, agregando que los países que más publicaban en Biología Tropical eran Costa Rica, México y los EEUU (Monge-Nájera \& Ho, 2016). En el periodo previo (2003-2012), México, Costa Rica y Venezuela eran las principales fuentes de publicaciones, con aumento anual de los artículos producto de colaboración inter-institucional y una media de tres autores por artículo (Filippo, Córdoba-González, \& Sanz-Casado, 2016).

En otro artículo se presenta una reseña histórica de la fundación y primera década de Cuadernos de Investigación UNED / UNED Research Journal (Seas, 2019). En este, analizamos las tendencias estadísticas de esta primera década, considerando cuatro temas: quiénes publican, qué publican, cómo han variado las tendencias a lo largo del tiempo y qué podemos esperar en el futuro.

\section{MÉTODOS}

Tabulamos toda la información de los artículos publicados desde los inicios de la revista en el año 2009 hasta el año 2018. Incluimos artículos científicos y notas cortas.

Generamos una base de datos con toda la información recopilada, y para garantizar la calidad de los datos, hicimos un proceso de normalización de las entradas de datos.

Analizamos año de publicación, volumen, título, idioma del artículo, área temática, cantidad de autores, género de los autores, afiliaciones, país de procedencia de los autores y país del sitio de estudio del manuscrito, cantidad de páginas, figuras y cuadros.

Ética, conflicto de intereses y declaración de financiamiento: los autores declaran haber cumplido con todos los requisitos éticos y legales pertinentes, tanto durante el estudio como en el manuscrito y que todas las fuentes financieras se detallan plena y claramente en la sección de agradecimientos. Asimismo, están de acuerdo con la versión editada final del documento. Por razones de transparencia y normas establecidas de la revista, al ser los autores miembros del cuerpo editorial no pueden participar del proceso de decisión, por lo que al final se indica el nombre del editor de este documento. El respectivo documento legal firmado se encuentra en los archivos de la revista.

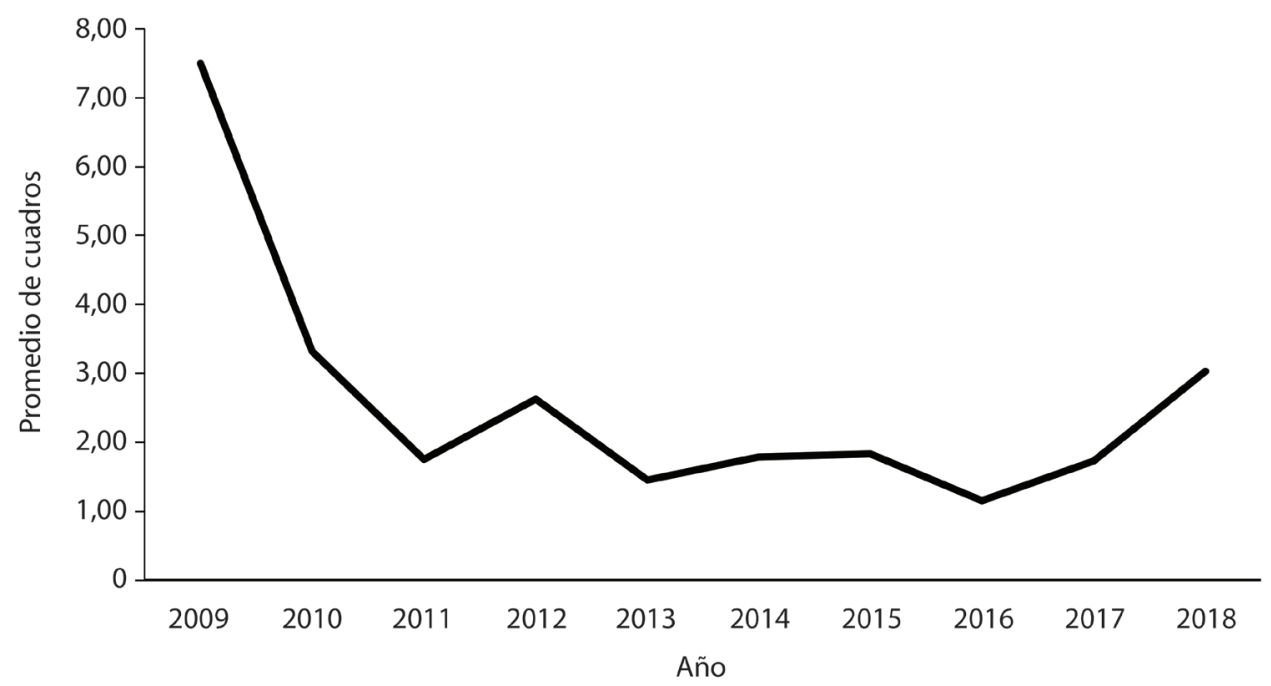

Fig. 1. Promedio anual de cuadros por artículo publicado durante los últimos diez años (2009-2018). 


\section{RESULTADOS}

La cantidad de cuadros por artículo fue alta al inicio, con un promedio superior a siete, pero del 2011 al 2018 ha sido mucho más bajo, oscilando de forma estable entre dos y tres cuadros por artículo (Fig. 1).

La cantidad de figuras por artículo oscila entre dos y cinco, sin otra tendencia al cambio (Fig. 2).

Los artículos han tendido a acortarse, desde un promedio de más de 16 páginas por artículo en 2009, a 8-9 después, con un ligero aumento a 12 páginas por artículo en 2018 (Fig. 3).
La cantidad de autores por artículo oscila entre dos y tres, con una ligera disminución en 2010 y un aumento a tres en 2012 (Fig. 4).

En el decenio, la presencia de autoras ha oscilado entre un tercio y la mitad, con mayor estabilidad al final del periodo (Fig. 5).

Las seis personas con mayor cantidad total de artículos publicados en el decenio (Fig. 6) son de la Universidad Estatal a Distancia (UNED), seguida de la Universidad de Costa Rica (UCR); la mitad de ellas, son mujeres.

La revista publica en todos los campos de la ciencia, sin embargo, predomina fuertemente la biología (Fig. 7).

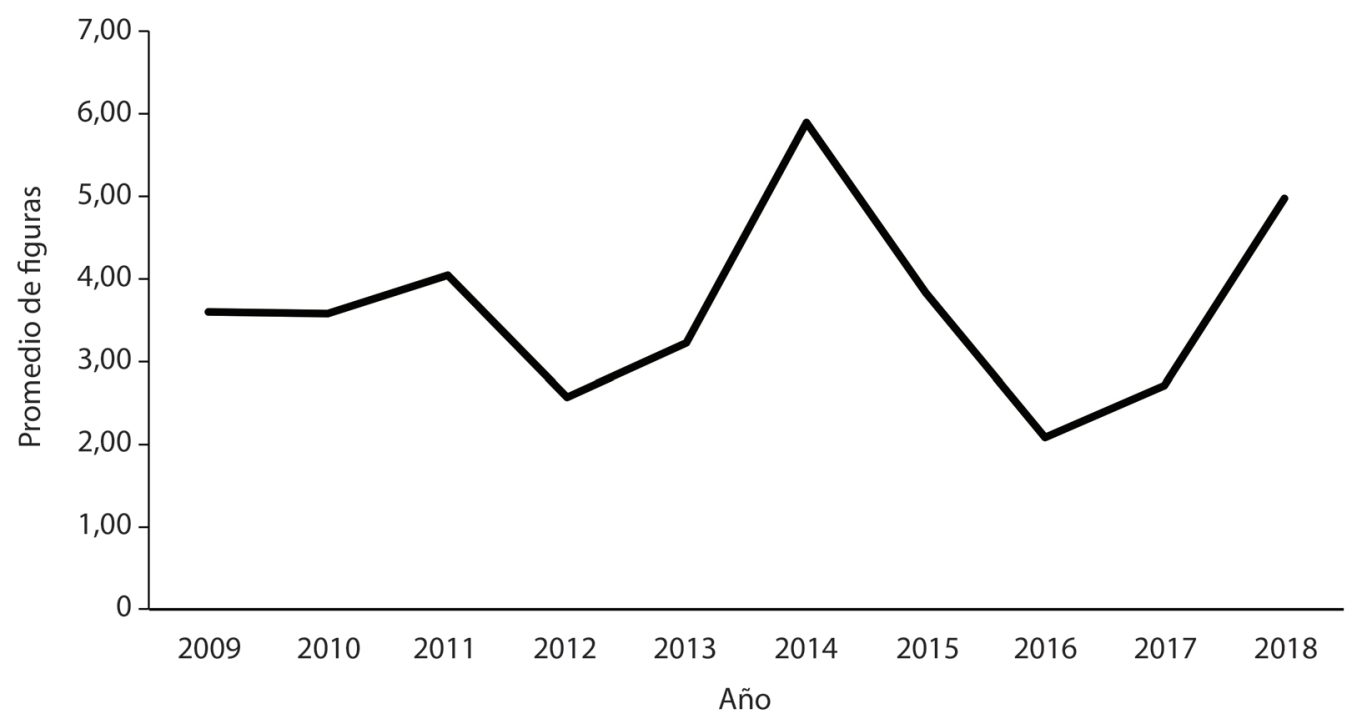

Fig. 2. Promedio anual de figuras por artículo publicado durante los últimos diez años (2009-2018).

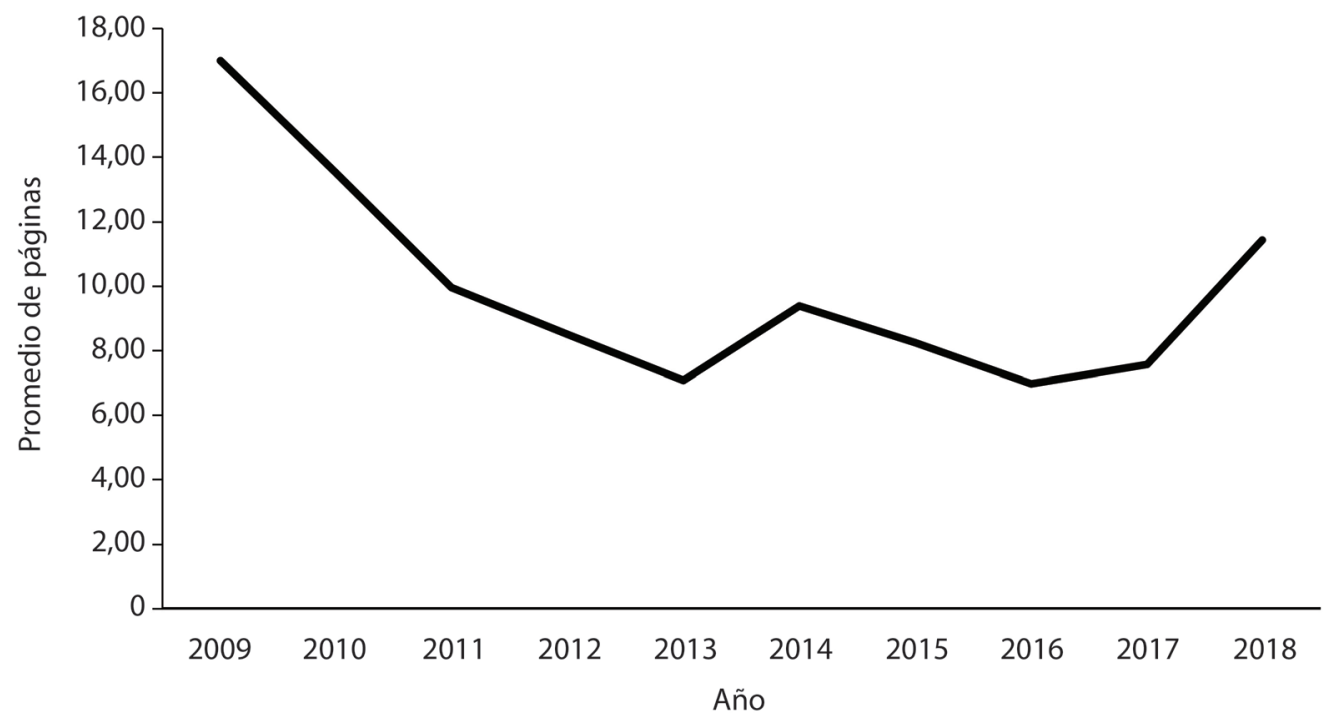

Fig.3. Promedio anual de páginas por artículo publicado durante los últimos diez años (2009-2018). 


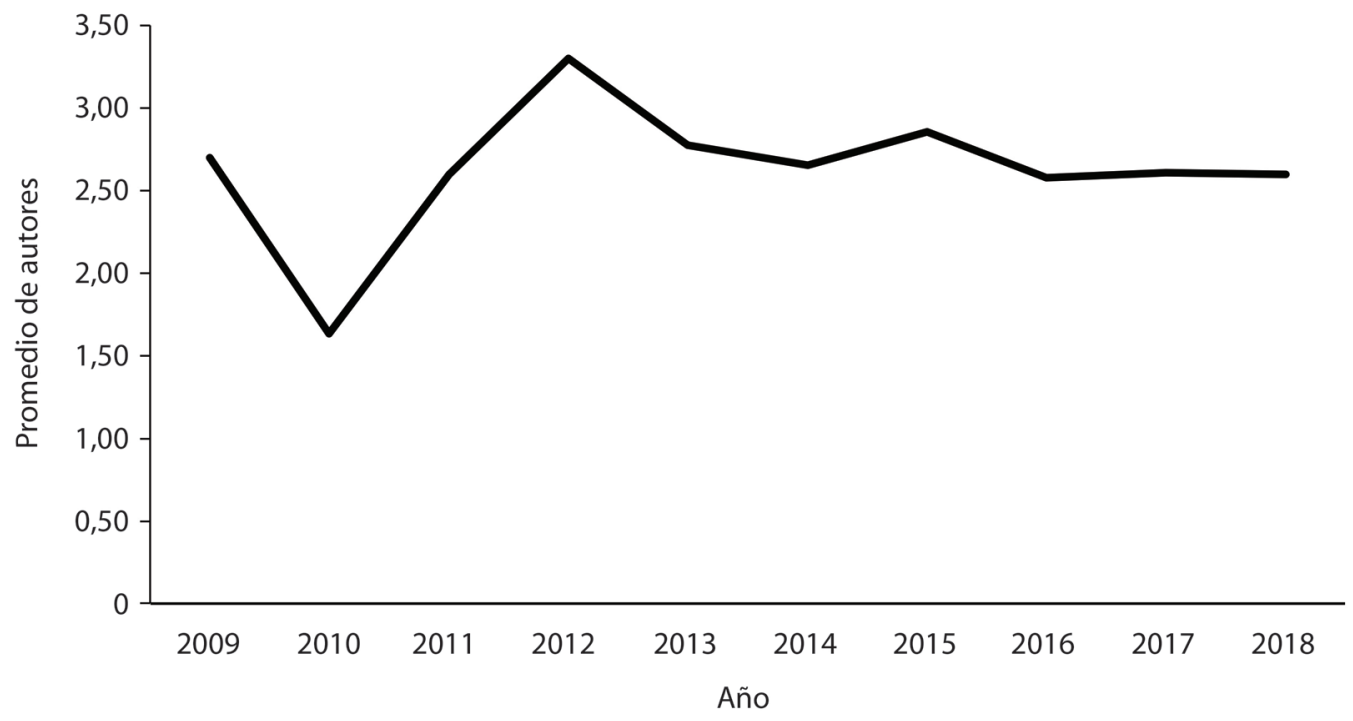

Fig. 4. Promedio anual de autores por artículo publicado durante los últimos diez años (2009-2018).

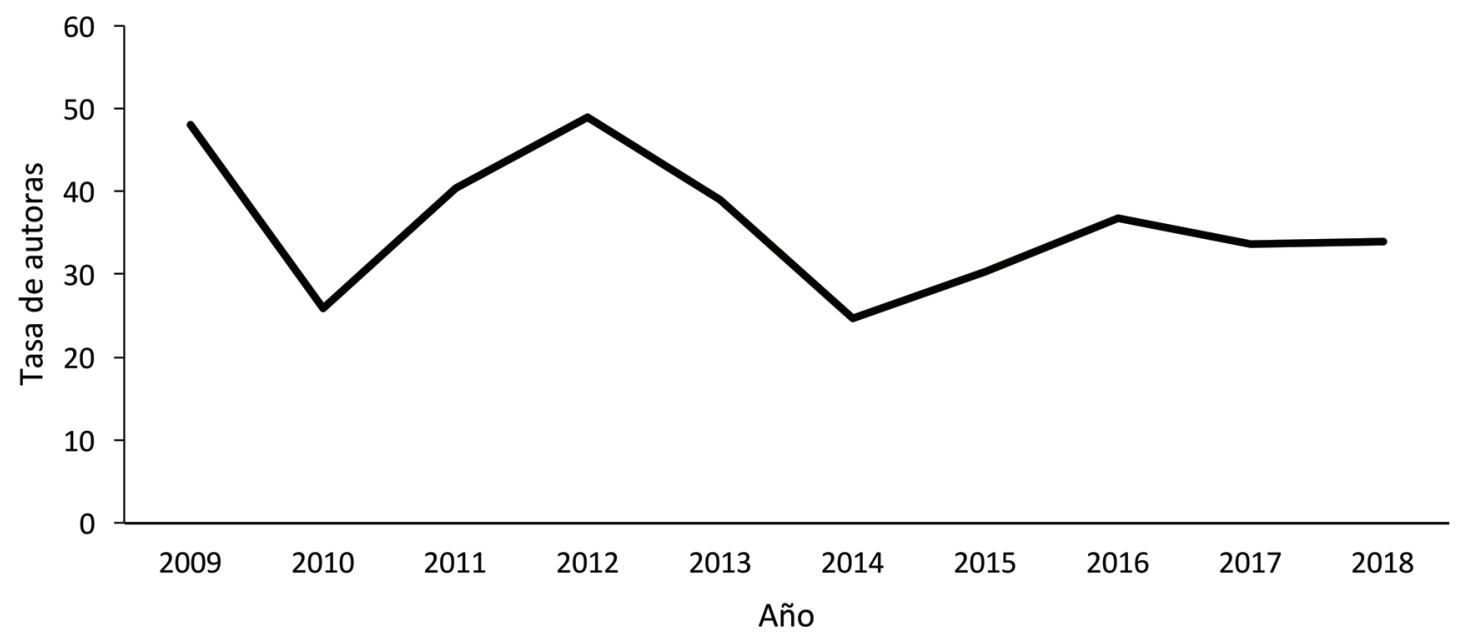

Fig. 5. Tasa de autoras de los últimos diez años de la revista (2009-2018).

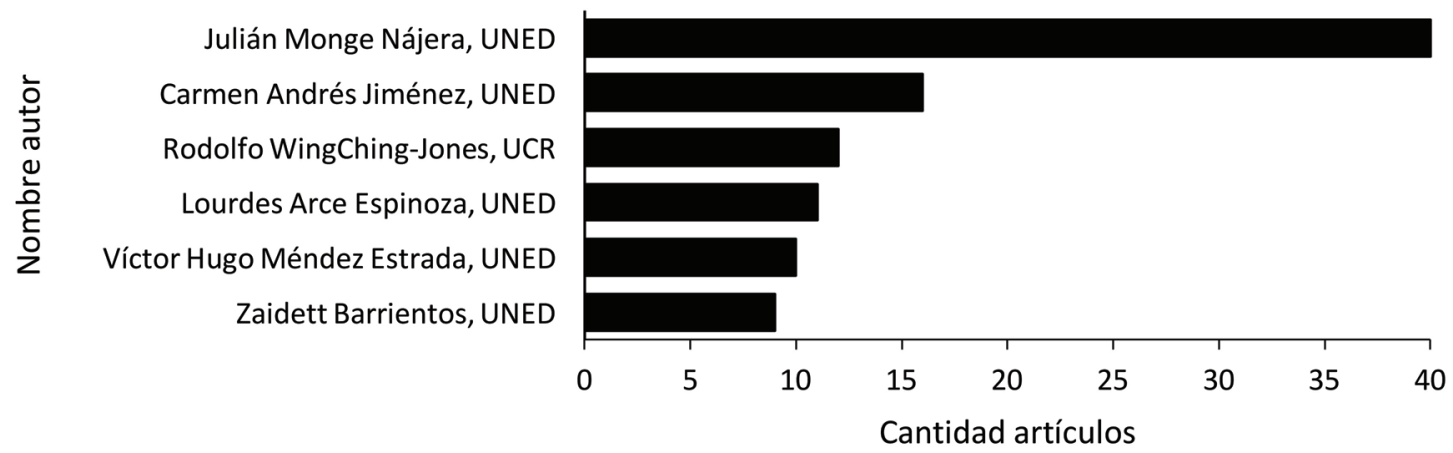

Fig. 6. Autores con mayor participacion en la revista en los últimos diez años (2009-2018). 


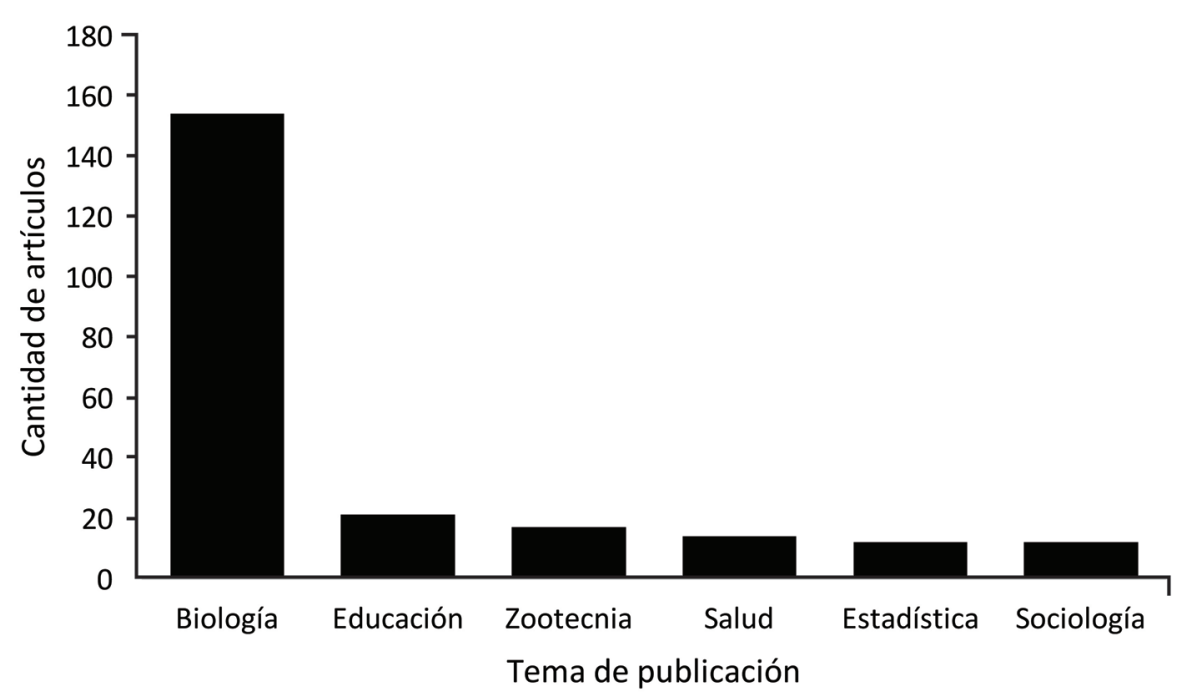

Fig. 7. Cantidad de artículos publicados por tema en el periodo 2009-2018.

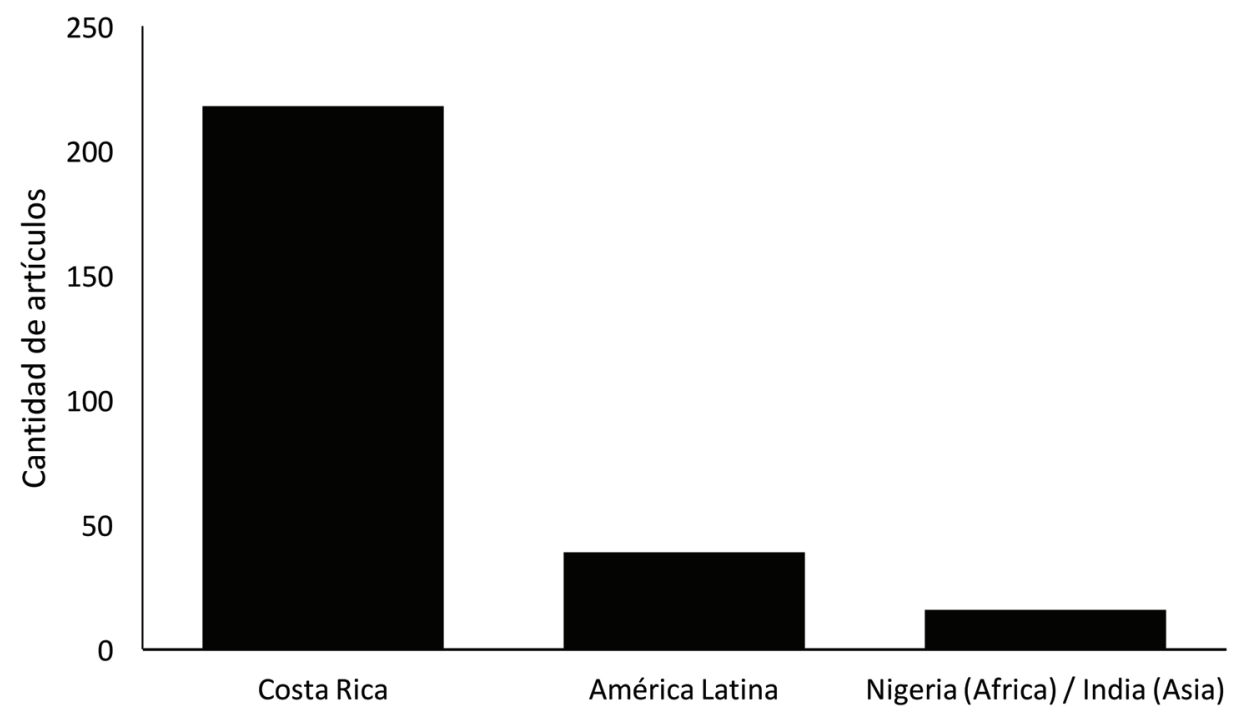

Fig. 8. Países estudiados dominantes en la revista (2009-2018).

En el apéndice 1 se muestra la lista completa de temas y la cantidad de artículos publicados en cada uno.

Geográficamente, predominan los artículos sobre Costa Rica, seguidos de lejos por el resto de América Latina, África y Asia (Fig. 8).

Históricamente, la cantidad de artículos provenientes de Costa Rica se ha cuatriplicado, México ha tenido grandes fluctuaciones (con un pico en 2013) y Argentina se ha mantenido baja pero constante (Fig. 9).

En cuanto al país de las afiliaciones de los autores, Costa Rica domina por mucho, seguida con números relativamente bajos por México, EEUU, Argentina y Nigeria (Fig. 10).

Las instituciones que predominan como fuentes de artículos son la UNED, UCR y la Universidad Nacional de Costa Rica (UNA). De lejos, le sigue la Universidad Nacional Autónoma de México, UNAM (y una investigadora independiente; Fig. 11).

Históricamente, el aporte de la UNED ha sido estable, excepto por un pico en 2018 que es resultado de la publicación de un fascículo especial producido por el 


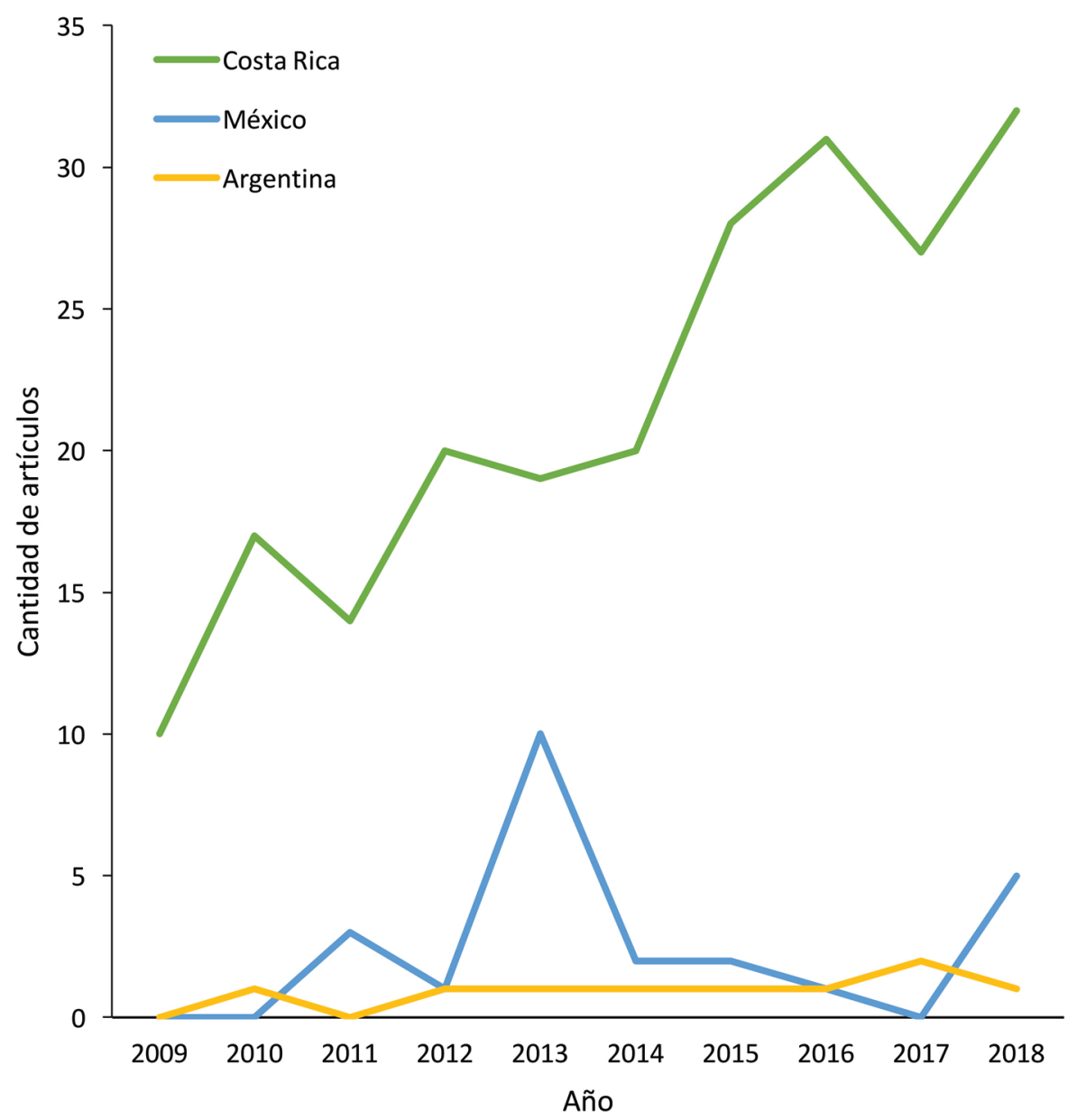

Fig. 9. Cantidad de artículos publicados en la revista por pais y año según sitio de muestreo.

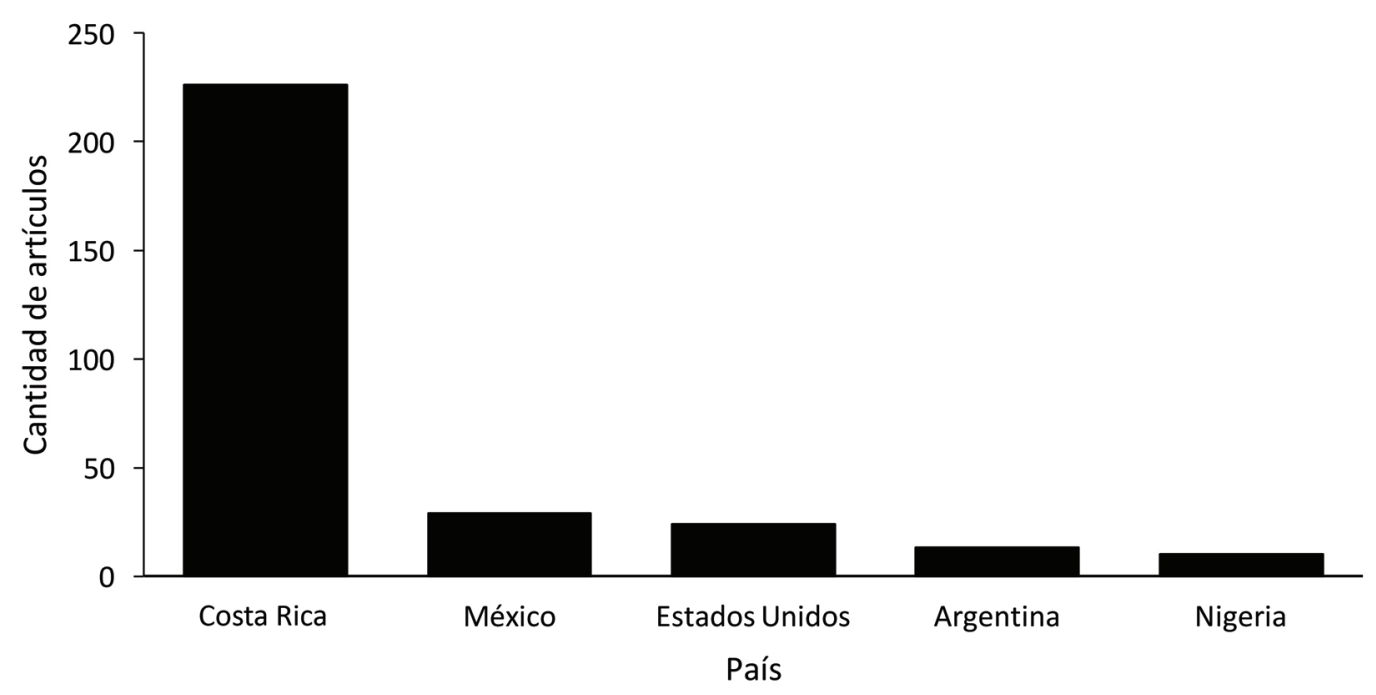

Fig. 10. País de afiliación del autor más publicados durante 2009-2018. 


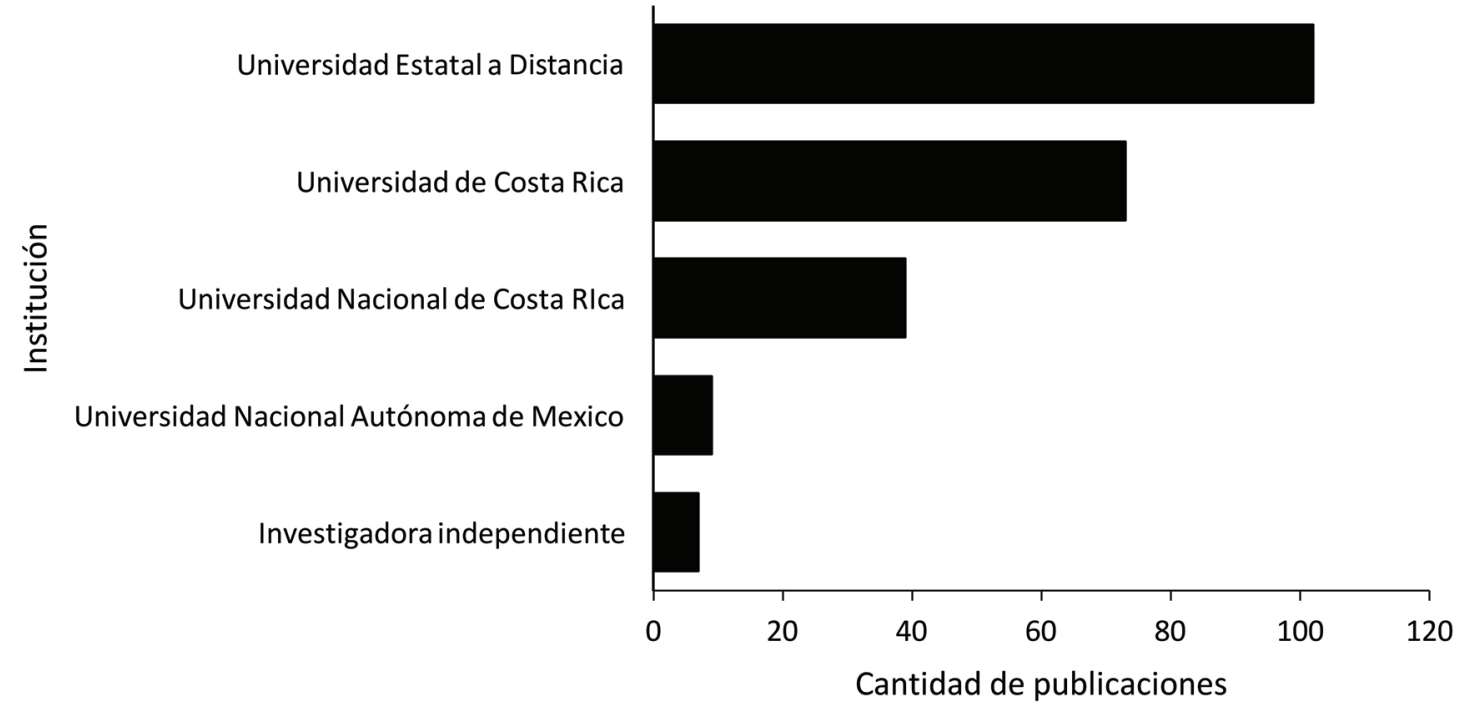

Fig. 11. Instituciones con más publicaciones durante 2009-2018.

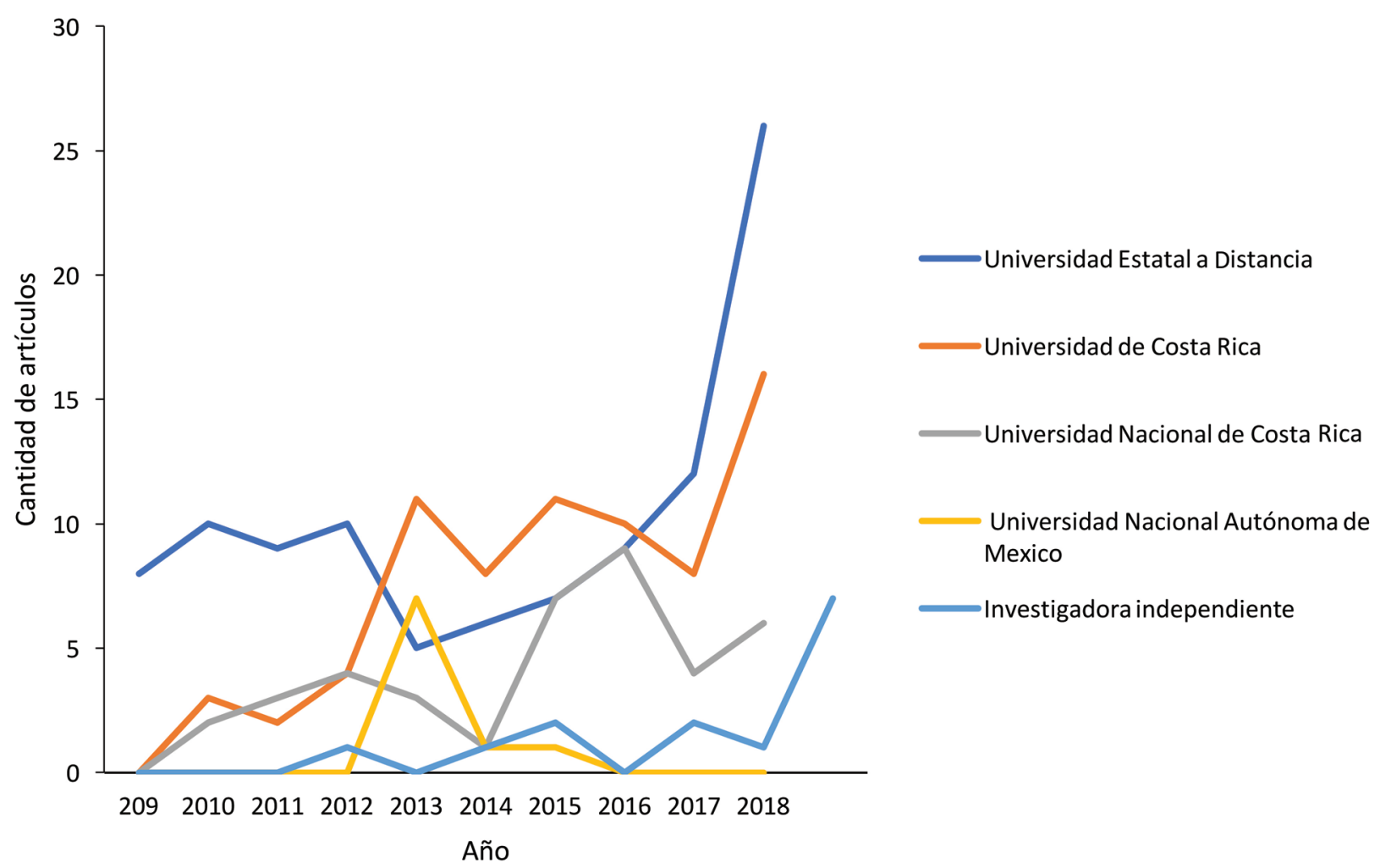

Fig. 12. Cantidad de artículos publicados en la revista por institución y año, entre los años 2009 y 2018.

Laboratorio de Ecología Urbana de la UNED. Sin embargo, también juega un papel fundamental la Universidad de Costa Rica, que en 2004 triplicó su aporte y cada cinco años parece aumentar. La tendencia general es al aumento, salvo en el caso de la UNAM, que históricamente ha disminuido (Fig. 12). 


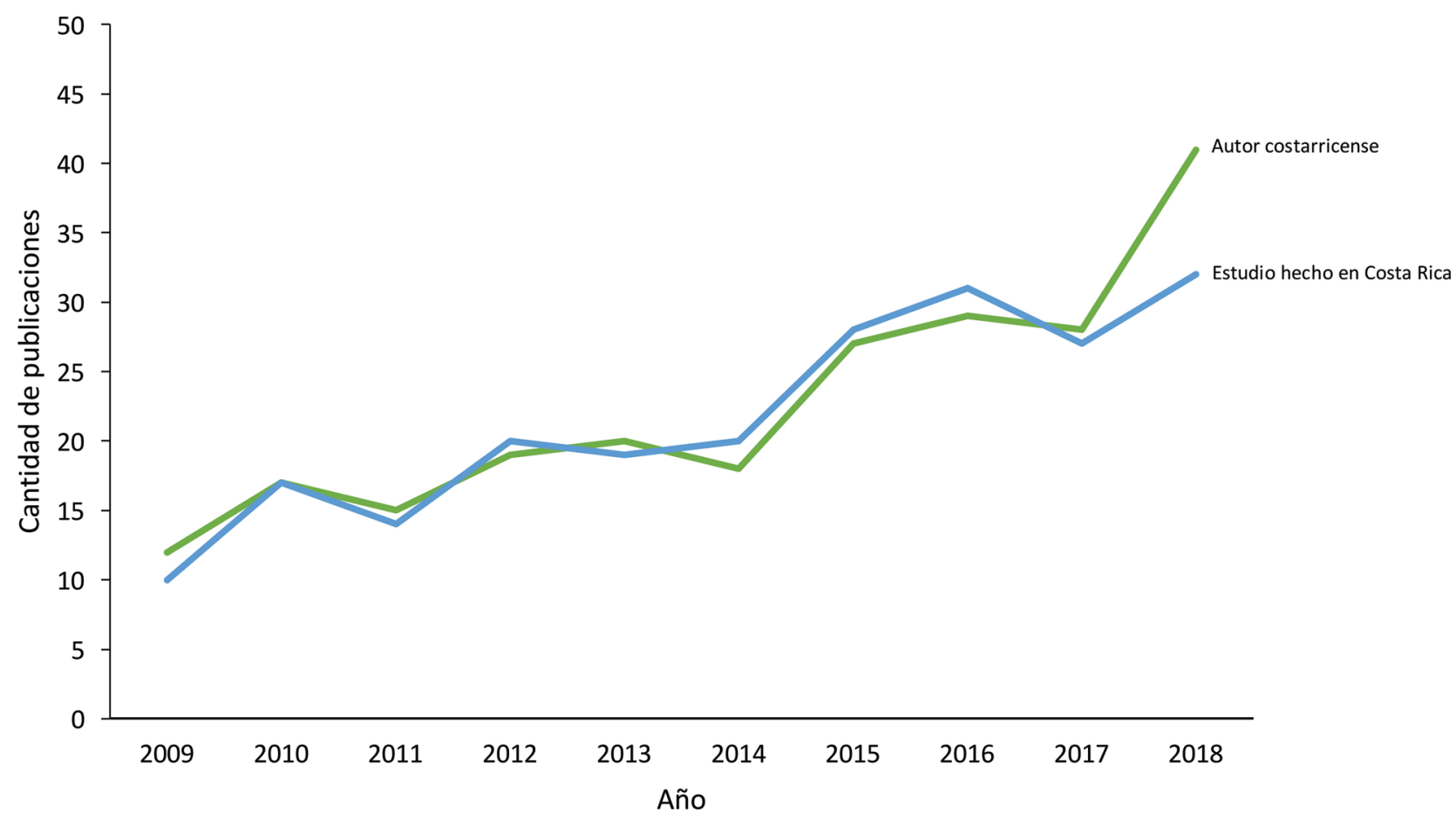

Fig. 13. Comparación anual entre la cantidad de artículos publicados en la revista por autores costarricenses y autores que hicieron su estudio en Costa Rica entre los años 2009-2018.

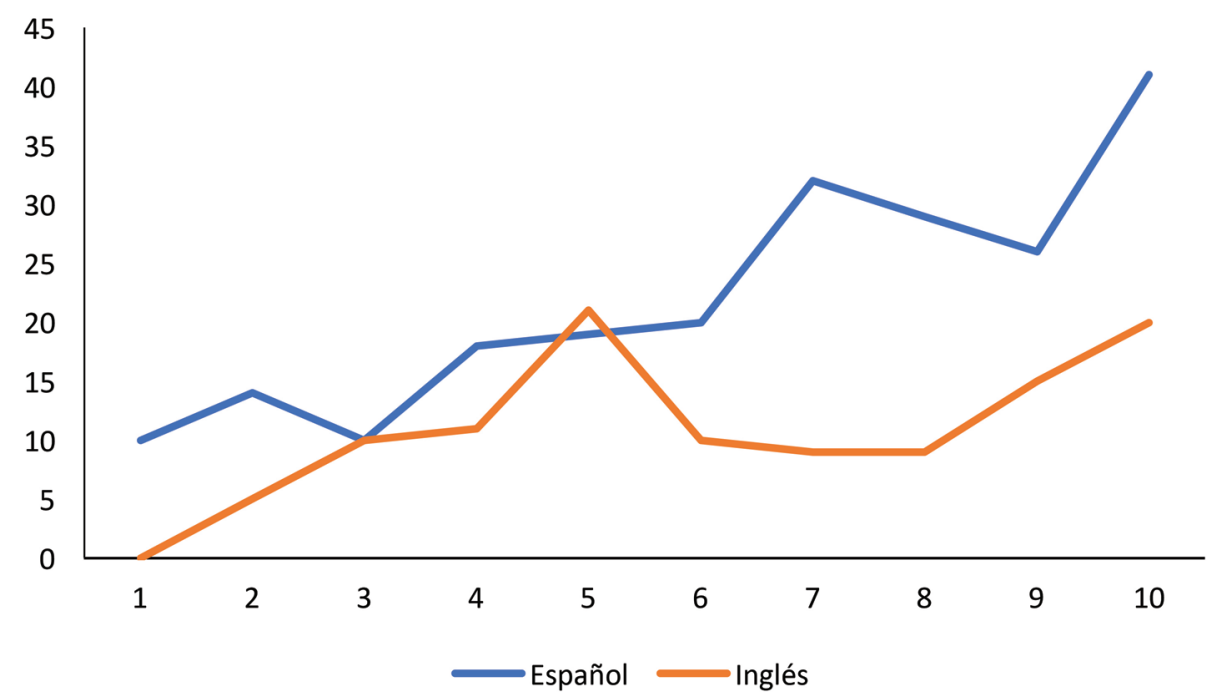

Fig. 14. Evolución del número de artículos según el idioma entre los años 2009 y 2018.

La correlación entre autores costarricenses, y estudios sobre Costa Rica, indica que en general, quienes publican sobre Costa Rica son autores costarricenses (Fig. 13).

Aunque tanto la cantidad de artículos en inglés, como la de artículos en español, están aumentado, el aumento de los artículos en español es más marcado y constante (Fig. 14).

\section{DISCUSIÓN}

El único estudio que analizó la cantidad de cuadros (tablas) por artículo en una revista científica costarricense fue el de Monge-Nájera y Díaz (1988), el cual halló un promedio de dos cuadros por artículo, similar al promedio actual de Cuadernos, posiblemente por la tendencia 
actual a estandarizar los artículos científicos en cuanto a extensión y contenido. En su etapa inicial, el promedio de cuadros por artículo fue mucho mayor, pero este promedio está sesgado por un trabajo publicado en el primer número de la revista; un trabajo excelente, que incluía 38 cuadros. Actualmente esto no se da, ya que se cuenta con la herramienta de archivos suplementarios para la información detallada, complementando el artículo, que así permanece conciso.

La cantidad de figuras por artículo en Cuadernos también es similar a la informada para la Revista de Biología Tropical hace tres décadas (Monge-Nájera \& Díaz, 1988). El que una revista relativamente pequeña y nueva se asemeje a otra tan establecida como Biología Tropical puede ser un indicador del seguimiento de procedimientos y normas internacionales, que redundan en la buena calidad de los documentos. Se incentiva al autor a que incluya solamente lo mas relevante para su estudio y que lo demás aparezca como material suplementario. La tendencia hacia artículos más cortos en años recientes también es similar a lo ya conocido y refleja mayor presión por parte de los editores en cuanto a la capacidad de síntesis de los autores (Monge-Nájera \& Díaz, 1988).

El hecho de que cada artículo sea preparado por entre dos y tres autores se encuentra dentro de lo normal en artículos científicos latinoamericanos y calza con la tendencia hacia realizar estudios más complejos y multidisciplinarios, que requieren cada vez más investigadores (Monge-Nájera \& Díaz, 1988; Nielsen-Muñoz et al., 2012; Filippo et al., 2016).

La presencia femenina en los artículos ha sido poco estudiada en revistas científicas costarricenses. En Cuadernos hay plena paridad entre los sexos, un poco mejor que en la Revista Costarricense de Psicología, donde las mujeres, aunque predominantes en el gremio de la psicología, solo representan un 40\% (Carvajal-Angulo \& Matamoros-Solís, 2012). Cuadernos publica una proporción dominante de artículos de ciencias naturales, por lo que la fuerte presencia femenina puede ser el reflejo de que en esta área ha habido un alto interés por incentivar a las mujeres, dado que en general las autorías femeninas siguen siendo insuficientes (Fine \& Shane, 2018). En Costa Rica la desigualdad es menor, por ejemplo, desde 1996 las mujeres predominan en la Escuela de Biología de la UCR (García, 2009).

La predominancia de autores de la misma institución que edita la revista, o sea, la UNED costarricense, es también un patrón conocido, por ejemplo, en la Revista de Biología Tropical, que es publicada por la UCR y -predeciblemente-- dominada por autores de esa institución
(Nielsen-Muñoz et al., 2012; Filippo et al., 2016; MongeNájera \& Ho, 2016).

La predominancia de estudios sobre Costa Rica, seguidos por otros de la región centroamericana y resto de América Latina, también es típica de las revistas científicas internacionales de Costa Rica, que tienen su mayor área de influencia en Mesoamérica; al igual que la ligera predominancia del español sobre el inglés, también típica de las revistas costarricenses (Monge-Nájera \& Díaz, 1988; Barrientos \& Monge-Nájera, 1990; Nielsen-Muñoz et al., 2012). Esta dominancia regional y lingüística no ha cambiado en décadas (Carvajal-Angulo \& MatamorosSolís, 2012; Filippo et al., 2016; Monge-Nájera \& Ho, 2016). La posible razón es que la revista fue creada con el fin de incentivar que las investigaciones de la misma UNED sean publicadas en ella, es la revista de la casa. Quizás sus documentos lleguen a ser más leídos por el alcance que tiene, pero siempre permanece el hecho de que las personas interesadas en estudios sobre temas costarricenses, trabajan principalmente en Costa Rica.

El crecimiento de la revista en cuanto a número de artículos es característico de las revistas jóvenes (MongeNájera \& Díaz, 1988) pero tras este periodo inicial el tamaño de cada fascículo varía poco (Filippo et al., 2016; Monge-Nájera \& Ho, 2016). Cuadernos puede estar alcanzando un límite debido a su capacidad en el proceso editorial, pero su crecimiento podría continuar si recibe mayor apoyo financiero. Aparte de lo financiero, la revista es básicamente lo que la hacen sus editores, y en este caso, el futuro de la revista seguramente reflejará cuan competentes y dedicadas sean las personas que la editen en el futuro.

En conclusión, los patrones históricos de Cuadernos coinciden con el de otras revistas científicas como Biología Tropical que, aunque publicadas en países pequeños, se insertan con éxito en el mundo mucho más amplio de la ciencia internacional.

\section{AGRADECIMIENTOS}

Agradecemos a Jeise Vargas Delgado por su ayuda con el registro y análisis estadístico de los datos.

\section{REFERENCIAS}

Barrientos, Z., \& Monge-Nájera, J. (1990). Los 40 Años de la Revista Turrialba: Un Análisis de los Artículos Publicados en ese Período. Turrialba, 40, 1, 1-4.

Bunge, M. (2007). Diccionario de Filosofía. Madrid, España: Siglo XXI Editores. 
Carvajal-Angulo, E., \& Matamoros-Solís, L. (2012). Análisis bibliométrico de la Revista Costarricense de Psicología, periodo 2001-2011. A propósito de los 30 años de su creación. Revista Costarricense de Psicología, 31, 1-2, 1-20.

Filippo, D. de, Córdoba-González, S., \& Sanz-Casado, E. (2016). Bibliometría de la colaboración e impacto de la Revista de Biología Tropical (Web of Science 2003-2012). Revista de Biología Tropical, 64(1), 147-156. DOI: 10.15517/rbt. v64i1.18241

Fine, I., \& Shen, A. (2018, 8 de marzo). Perish not publish? New study quantifies the lack of female authors in scientific journals. The Conversation. Recuperado de http:// theconversation.com/perish-not-publish-new-studyquantifies-the-lack-of-female-authors-in-scientific-journals-92999

García G., J. (2009). Breve historia de la Escuela de Biología de la Universidad de Costa Rica (1957-2009). Revista de Biología Tropical, 57(1), 1-14.

Monge-Nájera, J., \& Díaz, L. (1988). Thirty-five years of Tropical biology: a quantitative history. Revista de Biología Tropical, 36(2B), 347-359.

Monge-Nájera, J., \& Ho, Y.S. (2016). Bibliometry of the Revista de Biología Tropical / International Journal of Tropical Biology and Conservation: document types, languages, countries, institutions, citations and article lifespan. Revista de Biología Tropical, 64(3), 1223-1235. DOI: 10.15517/rbt.v64i3.22142

Nielsen-Muñoz, V., Azofeifa-Mora, A.B., \& Monge-Nájera, J. (2012).Bibliometry of Costa Rica biodiversity studies published in the Revista de Biología Tropical/International Journal of Tropical Biology and Conservation (20002010): the content and importance of a leading tropical biology journal in its 60th Anniversary. Revista de Biología Tropical, 60(4), 1405-1413. DOI: 10.15517/rbt. v60i4.2043

Seas, C. (2019). Reseña histórica. Los primeros diez años de Cuadernos de Investigación UNED: el recuerdo de los fundadores. UNED Research Journal, 11(2), 9-13.

\section{APÉNDICE 1}

CUADRO 1

Cantidad de artículos publicados por tema en el periodo 200-2018

\begin{tabular}{lc} 
& Nemámero de artículos \\
Biología & 154 \\
Educación & 21 \\
Zootecnia & 17 \\
Salud & 14 \\
Estadística & 12 \\
Sociología & 12 \\
Administración & 8 \\
Fitotecnia & 7 \\
Microbiología & 6 \\
Microeconomía & 6 \\
Química & 6 \\
Agronomía & 5 \\
Antropología & 5 \\
Psicología & 12 \\
Ingeniería & 5 \\
Nutrición & 1 \\
Recursos Humanos & 1 \\
Historia & 1 \\
Turismo & 1 \\
Politología & 4 \\
Bioestadística & 1 \\
Criminología & 4 \\
Informática & 3 \\
Medicina & 1 \\
Meteorología & 1 \\
\hline
\end{tabular}

\section{EDITADO POR FRANK GONZALEZ}

